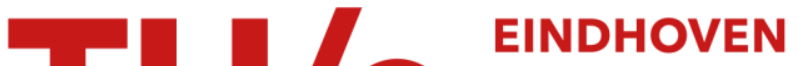 UNIVERSITY OF TECHNOLOGY
}

\section{A new design method for frequency-reconfigurable antennas using multiple tuning components}

\section{Citation for published version (APA):}

Bronckers, S., Roc'h, A., \& Smolders, B. (2019). A new design method for frequency-reconfigurable antennas using multiple tuning components. IEEE Transactions on Antennas and Propagation, 67(12), 7285-7295. [8777318]. https://doi.org/10.1109/TAP.2019.2930204

DOI:

10.1109/TAP.2019.2930204

Document status and date:

Published: 01/12/2019

\section{Document Version:}

Accepted manuscript including changes made at the peer-review stage

\section{Please check the document version of this publication:}

- A submitted manuscript is the version of the article upon submission and before peer-review. There can be important differences between the submitted version and the official published version of record. People interested in the research are advised to contact the author for the final version of the publication, or visit the $\mathrm{DOI}$ to the publisher's website.

- The final author version and the galley proof are versions of the publication after peer review.

- The final published version features the final layout of the paper including the volume, issue and page numbers.

Link to publication

\section{General rights}

Copyright and moral rights for the publications made accessible in the public portal are retained by the authors and/or other copyright owners and it is a condition of accessing publications that users recognise and abide by the legal requirements associated with these rights.

- Users may download and print one copy of any publication from the public portal for the purpose of private study or research.

- You may not further distribute the material or use it for any profit-making activity or commercial gain

- You may freely distribute the URL identifying the publication in the public portal.

If the publication is distributed under the terms of Article 25fa of the Dutch Copyright Act, indicated by the "Taverne" license above, please follow below link for the End User Agreement:

www.tue.nl/taverne

Take down policy

If you believe that this document breaches copyright please contact us at:

openaccess@tue.nl

providing details and we will investigate your claim. 


\title{
A New Design Method for Frequency-Reconfigurable Antennas Using Multiple Tuning Components
}

\author{
L.A. Bronckers, Student Member, IEEE, A. Roc'h, Member, IEEE, and A.B. Smolders, Senior Member, IEEE
}

\begin{abstract}
Frequency-reconfigurable antennas seem a viable solution to achieve better performance and increased flexibility for integrated mobile phone antennas. Due to the lossy nature of many tunable components, the use of multiple combined tuning components in a single design seems attractive, as it can distribute the current over the components. However, many combinations of individual tuning component settings can result in an acceptable input match, while they will vary significantly in terms of radiation efficiency. It is challenging to distinguish between these radiating and non-radiating tuning component settings during the design procedure. In this paper, we propose a method to determine the component settings with the highest efficiency at a desired operating frequency. The method uses a single full-wave simulation, which is combined with circuit-level calculations. We discuss how to apply the method in detail, and demonstrate its functionality with an inverted-L antenna sporting three tunable BST capacitors. It is shown that the method can successfully predict tuning component settings for high antenna efficiency over a 1.4 to $2.8 \mathrm{GHz}$ tuning band, with total efficiencies up to $35 \%$ and radiation efficiencies up to $50 \%$. The method can easily be applied to any desired antenna geometry.
\end{abstract}

Index Terms-Antenna design method, Antenna efficiency, Frequency-reconfigurable antennas, Small antennas

\section{INTRODUCTION}

5 G-and-beyond wireless communication systems promise higher data rates using millimeter-wave communications, while the sub $6 \mathrm{GHz}$ bands will remain a critical part of future communication networks as well [1]-[4]. One of the changes for this frequency range is the inclusion of many more bands and more flexible frequency allocation. However, in mobile devices the available real estate for antennas is extremely limited. The 5G case of the sub $6 \mathrm{GHz}$ bands in a mobile communications scenario implies a scattering environment, i.e. the radiation pattern of the antenna is not of primary interest. Thus, the main focus for this application can be placed on the antenna's efficiency at the frequencies of interest. According to the fundamental limitations for small antennas [5]-[9], usually known as the Chu-Harrington limit, an increasing bandwidth requirement given a fixed volume and directivity will result in a decreasing maximum achievable antenna efficiency.

The Chu-Harrington limit applies not only to the total efficiency $\eta_{\text {tot }}=P_{\text {radiated }} / P_{\text {available }}$, the ratio of the available and radiated powers, but also to the radiation efficiency $\eta_{\text {rad }}=P_{\text {radiated }} / P_{\text {accepted }}$, the ratio of the accepted and radiated powers. If one of these efficiencies is known along with the antenna's reflection coefficient, the other can be calculated, and the total efficiency can be brought closer to the radiation efficiency by improving the antenna's input match. Nonetheless, the radiation efficiency serves as an upper limit of the total efficiency that can potentially be reached using (adaptive) matching circuits. The Chu-Harrington limit (assuming the antenna it is electrically small and exhibits only a single mode within the VSWR bandwidth) may be expressed for the radiation efficiency as [10]:

$$
\eta_{\text {rad }} \leq \frac{f_{\text {center }}}{f_{\max }-f_{\min }} \frac{(k a)^{3}}{1+(k a)^{2}} \frac{s-1}{\sqrt{s}},
$$

where $f_{\max }$ denotes the frequency above $f_{\text {center }}$ where the VSWR is equal to $s$ (arbitrary value), $f_{\min }$ denotes the frequency below $f_{\text {center }}$ where the VSWR is equal to $s, k=\frac{2 \pi}{\lambda}$ with $\lambda$ the free-space wavelength, and $a$ is the smallest sphere containing the antenna's current distribution. Note that $\frac{f_{\text {center }}}{f_{\max }-f_{\min }}$ is the device's Q-factor if $s$ is chosen to represent the half-power threshold, while the term $\frac{s-1}{\sqrt{s}}$ accounts for the threshold used in determining the bandwidth. This equation presents an upper bound that depends only on the fractional (matched) bandwidth with respect to the antenna size. None the less, it should be noted that the many assumptions made in deriving (1), combined with the performance limitations of realistic tuning components, make the limit hard to approach: if the bandwidth is narrow and fixed, the maximum achievable efficiency rises in a quadratic fashion over frequency, quickly rising above $100 \%$. Yet it gives a useful qualitative view of the fundamental trade-off that has to be made.

The traditional approach to enable a mobile device to support a large number of bands has been to apply antennas that cover a large bandwidth. More recently, adaptive matching networks were added to the mix [11]. While this allows an increase of the useful operating range of a given antenna in terms of input match, and to compensate for userinduced mismatch, it does not tackle the more fundamental problem posed by the Chu-Harrington limit. On the other hand, aperture-tuned frequency-reconfigurable antennas [12][21] allow us to circumvent the Chu-Harrington limit, by having only a small usable bandwidth of the antenna itself at a time. If a different operating band is desired, the antenna tuning is adapted. In these aperture-tuned antennas the current distribution on the radiating element itself is changed, resulting in a more fundamental change than is achieved by adapting the antenna matching.

For these reasons, there has been a lot of interest in the field of aperture-tuned frequency-reconfigurable antennas recently. Overall, the possible methods to create a frequency- 
reconfigurable antenna can be divided in tuning using electrical components, tunable materials and mechanical tuning. The mechanical tuning methods [15], [22]-[28] have several properties in common: they can offer high performance, but, since they require physical displacement they are prone to wear, and they require a relative large volume. While this does not necessarily pose problems in all applications, it makes the methods unsuitable for applications in mobile handsets. Considering material tuning [14], [15], [23], [29], [30], integration into a such devices is challenging unless a compact, pre-designed component is available. This leaves electrical tuning methods as the most viable option for handset applications. This can be either switching (using MEMS switches [14], [15], [21], [23], [31], [32], p-i-n diodes [14], [15], [21], [23], [33]-[37], SOI/SOS [11], [20], j-pHEMT [20] or optical [14], [15], [21], [23]), using switches in banks with fixed components [38][40], or continuously tunable devices (semiconductor varactor diodes [15], [21], [23], [35], [39]-[43], BST tunable capacitors [11], [20], [44], [45] or MEMS [16], [18]). If it is desired to cover a large range of frequencies while maintaining a relatively small bandwidth, the continuously tunable devices are most desirable for handset applications. Despite the large interest in frequency-reconfigurable antennas in research, most papers present designs and are therefore particular to a single geometry. A versatile and widely applicable design method is needed for adoption in practical mobile devices.

Aperture-tuned antennas provide the option to increase the radiation efficiency, but they pose another challenge: the tuning components have parasitic losses, resulting in a lower radiation efficiency. This can be especially problematic for highQ antennas [46] (i.e. small bandwidths), where the antenna structure becomes highly resonant, resulting in high currents and therefore possibly a low radiation efficiency. This problem can be partly resolved by avoiding a high antenna $\mathrm{Q}$ while using high-Q components, e.g. RF-MEMS [16], [18], but these are relatively expensive. Therefore, for mobile applications it would be preferable to find a way in which e.g. semiconductor varactors [15], [21], [23] or BST capacitors [11], [20], [44], [45] can be used efficiently for antennas. One option may be to apply multiple tuning components, opening up the possibility to distribute the current through the tuning components, thereby lowering the effect of their losses. The circuit-level representation of this could e.g. be a set of capacitors in parallel, where their capacitance values add towards the total while their parasitic resistances decrease towards the total. In addition, the tuning range (the frequency range over which the antenna may be used) could potentially be increased by employing multiple tuning components. However, the presence of multiple tuning components also increases the risk of having local resonances with a good input match, that barely radiate if not taken into account in the design and component settings.

Recently, a generalized method was proposed to use coupled antenna elements with decoupling and matching networks connecting to their interfaces [47]. The reflections and couplings of and between the antennas were characterized using the S-parameter matrix, after which a tunable decoupling and matching network was derived with the goal to simultaneously achieve decoupling and matching. The antennas were not

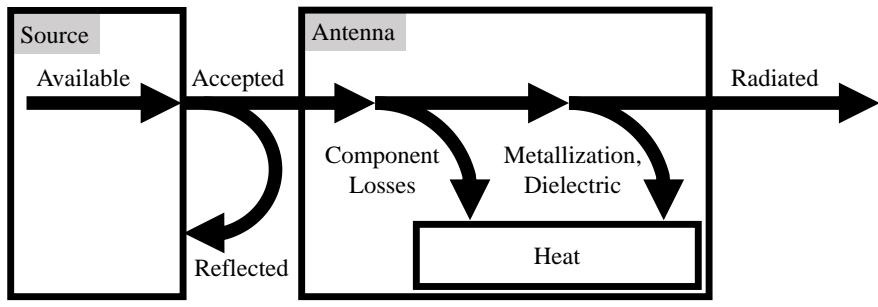

Fig. 1. Power flow for an aperture tuned antenna. The only parts not taken into account into the estimation of radiated power are the metallization and dielectric losses.

tuned in their aperture, wherefore only the total efficiency could be influenced while the radiation efficiency remained fixed. A somewhat similar concept was proposed in [48], where the transceiver was used to excite or receive the antenna elements with frequency-dependent weights. Again, since no tuning was performed in the antenna aperture, the radiation efficiency was not affected. So far, aperture-tuned frequencyreconfigurable antennas that were proposed have remained dependent on the geometry in their design method.

Thus, a good strategy is needed for the tuning component settings in aperture-reconfigurable antennas. A reconfigurable slot antenna using one [49] or two [50] varactors made use of the voltage distributions along the element for a desired mode to select the capacitor locations. More commonly, the choice of tuning element value and placement is made in stronger cohesion with the geometrical design [51], [52] or the components are placed at locations where a high voltage or current would exist when the antenna is operating without any (capacitive) loading [16], [53]-[55]. However, these approaches rely heavily on the geometry, and become increasingly complex as more tuning components are added. Moreover, they aim for maximum impact of the tuning component in terms of operating frequency, which does not necessarily result in a high efficiency. Therefore, a more general approach allowing one to make a distinction between the tuning component settings that are radiating well and those with a small efficiency is needed.

In this work, we introduce a design method to distinguish the tuning component settings resulting in high efficiency from those resulting in a low efficiency. Not only does it simplify the design process, but it provides a way to take advantage of using multiple tuning components, while avoiding settings that result in low antenna efficiencies. This will result in multiple configurations to cover a desired band from the input match perspective. Some of these will mostly excite non-radiating modes. However, by estimating the losses in the components we can then select the configuration that provides the highest (radiation or total) efficiency. We achieve this using just one single full-wave simulation, applying circuit-level calculations for the rest of the procedure. The method is independent on the antenna geometry, only requiring that the tuning components are the dominant loss mechanism in the antenna, in particular for the non-radiating modes.

While the method allows investigation on tuning component positioning as well as their settings, this work focuses on the tuning component setting aspects, as explained in the next section. We demonstrate the design method using an inverted- 


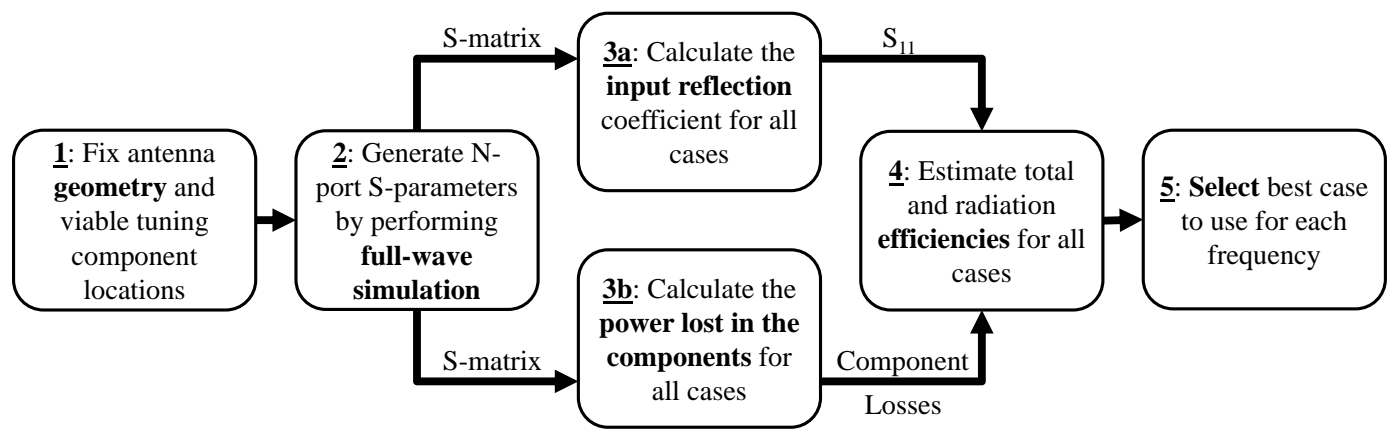

Fig. 2. Diagram showing the steps of the proposed design procedure. Steps 1 and 2 involve the geometry, which does not have to be considered. Step 3 uses circuit-level calculations, while the calculations in step 4 are simple additions and divisions which can be performed for a large set of cases. The estimated best case can then be selected in step 5 .

L antenna (ILA) geometry, as commonly found in mobile applications, and three commercially available tunable BST capacitors, in Section III. We then proceed with a realized design and present reflection coefficient and efficiency measurements, while explaining the results and proposing further improvements. The work is concluded in Section V.

\section{Design Method}

The key strength of our design method lies in its ability to largely decouple the tuning components settings from the geometrical design. Once the geometry is fixed, only simple (and therefore fast) calculations have to be performed. The goal is to find the component settings that will deliver us with the highest achievable (total or radiation) efficiency given a certain geometry with possible tuning component locations, using multiple tuning components.

In order to achieve this, we will first look at the power flow of the antenna, illustrated in Fig. 1. From the available power, part will be reflected at the antenna input. Since this power will not be radiated by the antenna, we model it as a loss towards the source. What remains is the accepted power, which can go three ways: it can be radiated (desired), dissipated in tuning components (not desired) or dissipated in metallization or dielectric (not desired). The metallization and dielectric losses are time-consuming to estimate, since they would require an electromagnetic model or an equivalent network for each excited mode. The tuning components potentially change the current distribution, wherefore a computationally expensive calculation would have to be performed to each potential combination of component settings. However, we will assume that for tunable antennas the metallization/dielectric losses are not independent from the component losses: high currents in the metallization or high fields in the dielectric will coincide with high currents through the tuning components, if the tuning component has a significant impact on the antenna performance. In addition, the radiation efficiency can be expected to be fairly high for most designs not incorporating tuning components, i.e. the untuned radiating mode has a high radiation efficiency. Under these assumptions, we can estimate the radiated power by neglecting metallization/dielectric losses and use this estimate to select the most optimal component settings. From this we can then estimate $\eta_{\text {rad }} \approx P_{\text {radiated }} / P_{\text {accepted }}$ and $\eta_{\text {tot }} \approx P_{\text {radiated }} / P_{\text {available. Note that this assumption creates }}$ an upper bound on the efficiencies, so we can identify the badly (or non) radiating modes with confidence.

Under these assumptions, we can model the antenna structure using only its S-parameter matrix. This can be viewed as an equivalent circuit for the antenna, and contains all relevant information to calculate the voltages and currents if the terminations on all ports and a source are defined. Note that, since we calculate the full S-parameter matrix, we also account for the coupling between the ports/components. In principle, the S-parameter matrix is an exact representation for the structure, limited only by the accuracy of the fullwave solver. So, if we obtain the S-parameter matrix with ports in the locations of all (potential) tuning components, we can calculate the voltages and currents at the ports for any tuning component and/or setting. This calculation does not require us to make any assumptions on the potentials of the ports with respect to one another, or their coupling - it only requires that the antenna is linear, i.e. that no harmonic frequency components are generated. Therefore the voltages and currents at the ports are identical to those calculated by CST microwave studio. Provided that the losses are included in the tuning component impedance model, we can also calculate the dissipated power in each of them. The proposed method is based around this principle, and employs it by calculating the dissipated power in the tuning components for a large set of possible tuning component settings, e.g. 32 possible settings each for three components (resulting in $32^{3}=32768$ cases).

By combining the S-parameter matrix along with circuit models for the tuning components, we can calculate the resulting input reflection coefficient of their combination. In addition, we can calculate the voltages and currents at each of the tuning component terminals, and therefore the power dissipated in each of them for a given setting. Since we know the available and accepted powers, we can then estimate an upper limit for the total and radiation efficiencies. This upper limit would be the exact (computational) efficiency if there are no thermal losses in the structure itself (e.g. metallization or dielectric losses). Thus, the estimate becomes more accurate for low-loss dielectrics and metallizations, as well as low-Q tuning components. Repeating the estimation for the efficiencies for all cases, we can then use these estimations to select the 'best' cases from the efficiency point of view. 
A high-level view of the proposed design method is given in Fig. 2. Starting on the left, it consists of the following steps:

1) Choose a geometrical design and potential tuning component locations. The rest of the procedure does not adapt the geometry, so it is crucial to carefully consider this step before proceeding. For instance, if tunable capacitors are used in parallel to the radiating element, consider that the operating frequency of the un-tuned mode will only shift down in frequency. In addition, avoid high-Q resonances in the geometry when no tuning components are placed.

2) Generate the S-parameter matrix over the entire desired frequency range for the chosen geometry using full-wave simulations. For the excitation and all potential capacitor locations, ports should be placed. So if we have $N_{\text {comp }}$ potential capacitor locations, we have $N=N_{\text {comp }}+1$ ports and therefore an $N \times N \times N_{\text {freq }}$ S-parameter matrix for $N_{\text {freq }}$ frequency points, capturing all the reflections and couplings when the components are all set to $50 \Omega$. This is all the information about the geometry we will use from this point on. The process up to this point is (computationally) relatively time-consuming, but has to be performed only once for a given geometry (and should be repeated if different tuning component locations are desired). All the steps that follow will use the $\mathrm{S}$-parameter matrix generated in this step.

3) Steps 3 and 4 will be performed for a large set of possible tuning component settings. First, we discretize the range of tuning component settings in suitable steps (e.g. 32 steps for each capacitance). Each combination (32768 for three capacitors with 32 steps each) of the capacitor settings will be called a case. Knowing this, along with a circuit model for the tuning components, we can calculate the input reflection coefficient for each case from the S-parameter matrix (step 3a), as well as the power that is dissipated in each of the tuning components (step 3b). This is done by converting the S-parameters to $\mathrm{ABCD}$, calculating the ABCD parameters of the tuning components, and cascading them. In this way, all the couplings are accounted for, and the resulting voltages and currents at the ports are identical to those that would be obtained using a 3D full-wave simulation with these component settings and models.

4) Knowing the input reflection coefficient, accepted power and power dissipated in the tuning components, we can now estimate the total and radiation efficiencies for each case. This is done as a function of frequency, so we end up with matrices containing estimated radiation and total efficiencies as a function frequency.

5) Now that we know the estimated efficiencies over frequency for each case, we can select the best setting for each frequency point by finding the maximum estimated total efficiencies. Note that it would be quite simple to also include bandwidth requirements in this step, including non-contiguous bands, or a required impedance range and look for the maximum radiation efficiency.

This procedure allows us to extract the most crucial informa- tion about the geometry and its electromagnetic behavior using a single full-wave simulation. It can be viewed as extracting a circuit-level model from the geometry using its S-parameter matrix, which is then used to identify how the tuning components will couple and what their losses will be, as well as to calculate the complete structure's input impedance. This is the key step of the procedure: using the tuning component models to calculate their dissipated powers, which is then used to estimate the antenna efficiencies. For situations incorporating a large number of tuning components, an optimization algorithm might be placed instead of steps 3, 4 and 5 to arrive at optimum solutions without calculating all possible cases. However, for the purpose of better demonstrating the design principle, we chose to calculate all cases, which could be completed within hours on a laptop for three tuning components with 32 steps each. Note that the amount of possible cases increases drastically for the amount of tuning components, so for e.g. six tuning components it would be desirable to implement an optimization algorithm instead. This does not change the strategy of the design procedure. The assumptions on which the procedure is based, and the procedure from an antenna point of view, remain unchanged. If the optimization algorithm works properly (i.e. it finds the global optimum) the result of step 5 will be unchanged. During the design process, the results of steps $3 \mathrm{a}$ and 4 are of particular interest, as these provide insight into the antenna's input match and estimated efficiencies. In the next section we will follow the procedure for a practical design.

\section{DESIGN USING BST CAPACITORS}

In order to demonstrate the design method, we have performed the complete procedure using an ILA geometry, as commonly applied in mobile devices, with six potential capacitor locations (out of which three will be used). We target a tuning band of 1.4 to $2.8 \mathrm{GHz}$ in order to cover a large number of LTE [56] and 5G NR [57] bands, GPS, and the $2.4 \mathrm{GHz} \mathrm{WiFi}$ bands.

Micro-electromechanical system (MEMS) devices can be found as continuously tunable capacitors, or as inter-digital capacitor arrays which are switched arrays that provide a range of capacitances. While they have a high quality factor, high linearity and low power consumption, they are relatively costly and have a lower reliability than solid-state devices [15], [23], [38], [44], [58]. Varactor diodes on the other hand provide fast switching at low cost with a long lifetime, but have a lower linearity and relatively low quality factor [15], [21], [23]. Barium-Strontium-Titanate (BST, a paraelectric material which changes its permittivity when a DC electric field is applied) based varactors offer a compromise between these two options, with a long life time, moderate quality factor and linearity, fast switching and low cost [11], [20], [44], [45]. Therefore this seems a very promising technology to use for frequency-reconfigurable antennas. The tuning components we will use are BST varactors with a tuning range of $0.61-3.2 \mathrm{pF}$. The selected components are ST Parascan STPTIC-27L2, in a WLCSP $0.4 \mathrm{~mm}$ package [45]. These components have four pins of which three are used: two RF 
ports (between which the capacitance is seen) and a DC bias pin, which uses one of the RF ports as a ground reference. We use a simple model for their behavior: a resistor in series with a capacitor. Lacking more complete information about the tuning component's parasitics and behavior, for each capacitor tuning setting the resistance (kept constant over frequency) is estimated to obtain a $Q=50$ at $2 \mathrm{GHz}$, corresponding to a decreasing $\mathrm{Q}$ over frequency.

The chosen geometry is shown in Fig. 3. We have chosen an inverted-L antenna (ILA) design since it is commonly found in smartphones, and allows for multiple tuning components to be placed in parallel along its length. Simplified views of the intended DC and RF connections are shown in Fig. 4. Current is allowed to flow over the ground plane along the ILA element virtually without interruption due to the presence of metallization on both front and back, connected using vias. The length of the radiating element (on the left of the front view in Fig. 3a) is only $20 \mathrm{~mm}\left(\approx \frac{\lambda}{10}\right.$ at $\left.1.4 \mathrm{GHz}\right)$, at $3.7 \mathrm{~mm}$ distance to the board ground. It is fed by a coplanar waveguide with ground (GCPW) transmission line to allow for easy mounting of tuning components along the feed line while avoiding radiation from that area, and an economic isola i-tera substrate is chosen. The board can accommodate a total of six tuning components (hence there are seven ports), with four of them on the radiating element and two of them on the GCPW feedline. The bias feeding lines (seen in the back view of Fig. 3b), with a gap to place an 0402 SMD-package inductor in the fabricated antenna, are included in the design, but the simulation is performed with a continuous ground plane to limit computation time. Due to the large amount of vias connecting the top and bottom ground planes, this is deemed a reasonable approximation, that saves simulation time and memory, allowing for a better meshing. The mounting area of a tuning component, with a bias line running on the back of the board from the bottom-right pin, can be seen in Fig. 3d. The spacing between the BST capacitor's connections is just $0.4 \mathrm{~mm}$. We now obtain the 7-port S-parameter matrix using the frequency-domain solver of CST Microwave Studio, which is then exported to Matlab, completing steps 1 and 2.

For this work, the possible port locations have been tested alternatively with various capacitance values in order to make a pre-selection for the best tuning component locations from an input match point of view. These experiments showed that the best results could be obtained using the three locations at the end of the radiating element. These are the locations that are closest to the location of the single tuning component that is traditionally used, employing the voltage maximum of the fundamental mode at the end of the radiating element. For the rest of this work, we will use these three locations. Using an optimization algorithm, this process may be automated as well, while maintaining reasonable calculation times.

The number of steps taken per component is a trade-off between the resolution achieved for the component settings and computational time. Here, the settings of the three components are swept in 32 steps each, resulting in 32768 cases, as shown in Fig. 5. The capacitor at the end of the element (C1) is changing fastest, i.e. is swept completely for the first 32 cases, while the second one is swept completely over the

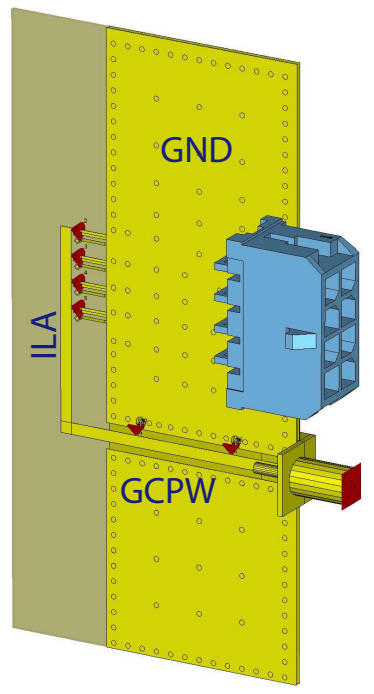

(a)

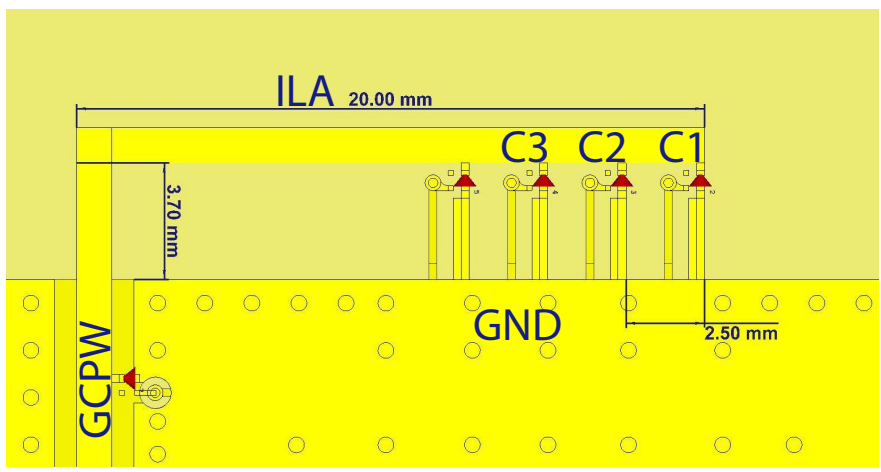

(c)

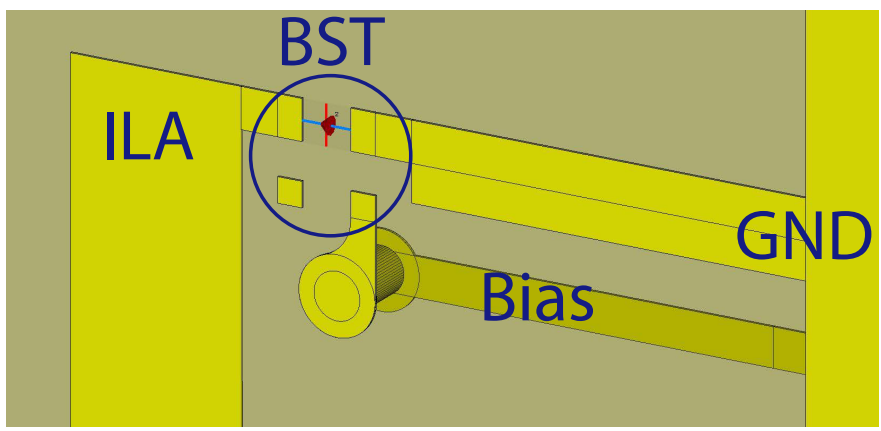

(d)

Fig. 3. Antenna geometry in (a) front view, (b) back view, (c) front view with dimensions and (d) close-up of BST mounting area (front), with the substrate semi-transparent. Possible capacitor locations are visible as discrete ports (red cones). In the bias lines on the rear side of the board inductors will be mounted. The ILA length is $20 \mathrm{~mm}$, and has a $3.7 \mathrm{~mm}$ distance to the ground. The BST capacitors at the end of the radiating element are placed $2.5 \mathrm{~mm}$ apart.

first 1024 cases, etc. All component values are swept from their smallest to their largest value for step 3, which can now be performed.

The results of step 3a are shown in Fig. 6, where we have plotted the frequencies where a match better than $-10 \mathrm{~dB}$ is achieved for all cases. As can be seen, there are a multitude of possibilities to cover the $1.4-2.8 \mathrm{GHz}$ range in terms of 


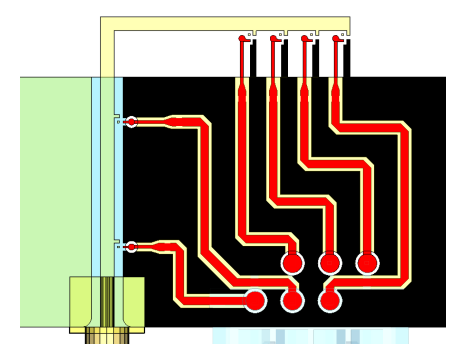

(a)

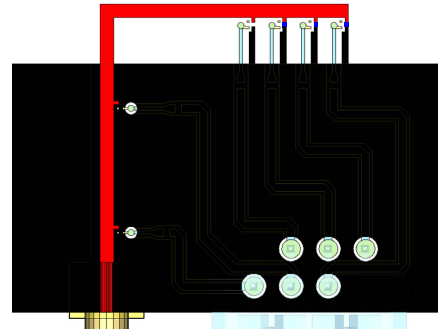

(b)
Fig. 4. Simplified views of the DC (a) and RF (b) connections of the antenna. For DC, the capacitors' ground connection is shown in black, with the bias voltage lines in red. For RF the ground plane (which will have potential differences due to the high frequency) is shown in black, with the ILA element indicated in red.

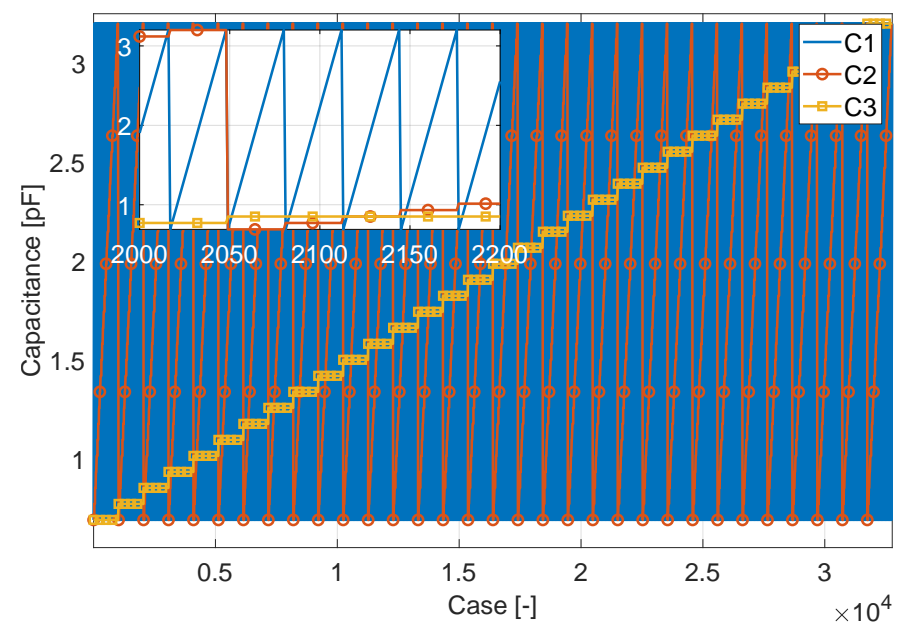

Fig. 5. Capacitor values as a function of case. $\mathrm{C} 1$ is the component at the end of the radiating element, counting from that point inwards. The inset shows a detail for cases 2000 to 2200 .

input match. It is interesting to see that, due to the way the capacitors were swept over the cases, the operating frequency decreases for increasing case number (i.e. more total capacitance). However, as discussed earlier, a matched antenna does not guarantee a well radiating antenna, especially with tuning components operating at a fairly low Q. Nonetheless it is desirable to verify these results at this stage of the procedure, since it may be required to adapt the geometrical design to achieve a good input match over the entire desired operating band, even accounting for all possible tuning component combinations, or to choose a tuning component with a different range. For instance, in this design it would have been possible to place components on the GCPW feedline as well or to use BST capacitors with a different capacitance range.

The results of step $3 \mathrm{~b}$ are not shown separately, as they do not give a useful estimate by themselves. Instead, we have plotted the estimated radiation and total efficiencies from step 4 in Fig. 7. In these figures, a lighter color corresponds to a higher efficiency. The combination of these figures, especially with Fig. 6, can provide some interesting insights. First, the estimated total efficiency never peaks above $25 \%$, while the estimated radiation efficiency peaks at nearly $100 \%$. The design procedure's goal here is to find the cases that are the

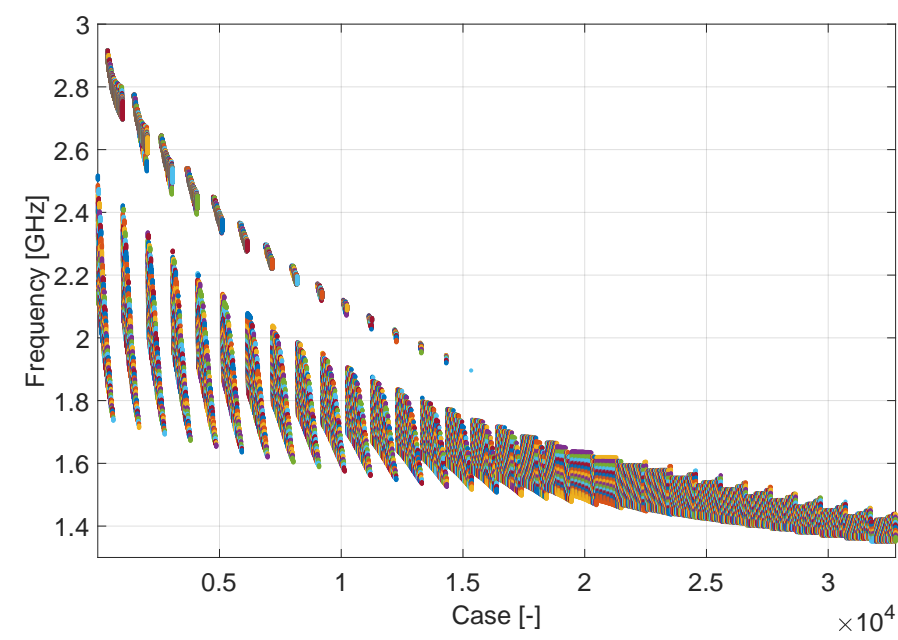

Fig. 6. Matched $\left(\left|S_{11}\right| \leq-10 \mathrm{~dB}\right)$ frequency ranges as a function of case number. Different colors are used to be able to distinguish the results for neighboring cases (possible tuning component setting combinations). In terms of input match, the desired $1.4-2.8 \mathrm{GHz}$ can be easily covered.

best compromise on this that can be made in terms of total efficiency. It is noteworthy that, referring to Fig. 1, we can only over-estimate the efficiency due to the approximation we make in neglecting the metallization and dielectric losses. Thus, we can identify the cases exhibiting low efficiencies with confidence. While Fig. $7 \mathrm{~b}$ mostly shows total efficiencies close to 0 , allowing us to discard a large number of cases, there are some cases with an estimated total efficiency of up to $25 \%$. Given the low $(\mathrm{Q}=50)$ quality factor of the components, compact dimensions, and the absence of a matching network, this is considered to be an acceptable result for the purpose of this demonstration, as these are the cases we are looking for.

Using the method, we are able to predict this behavior, and can choose the tuning component settings accordingly by selecting for maximum estimated total efficiency (step 5). We can then arrive at the voltage settings for the components using their voltage-capacitance relationship [45]. This is what we did for desired frequencies from 1.4 to $2.8 \mathrm{GHz}$, as shown for center frequencies spaced $100 \mathrm{MHz}$ apart in Table I, along with their respective estimated efficiencies. Note that settings can be obtained for any frequency in the range, and are not limited to the center frequencies shown in Table I. It can be observed that for the bands up to $2.3 \mathrm{GHz}, \mathrm{C} 2$ (the capacitor in the middle) is tuned as low as possible, while it is used as the main tuning element above that frequency (low capacitance values for $\mathrm{C} 1$ and $\mathrm{C} 3$ ). It would be hard to arrive at these settings without the proposed method. Next we verify the method by implementing the design and measuring its efficiencies using the settings from Table I, as discussed in the next section.

\section{Realized Design with BST CAPacitors}

The design is manufactured and the BST capacitors are placed, along with inductors in the bias feed lines. The realized, fully integrated, board is shown in Fig. 8. In particular the BST capacitors require specialized equipment, as the pitch between balls underneath the BST components is just $0.4 \mathrm{~mm}$. Finally, the SMA connector (for the RF signal) and a connector 


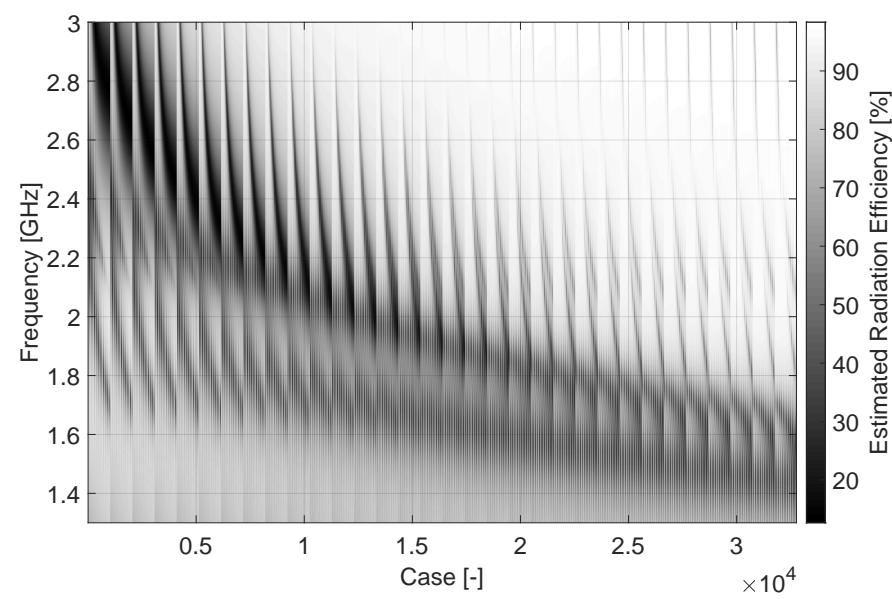

(a)

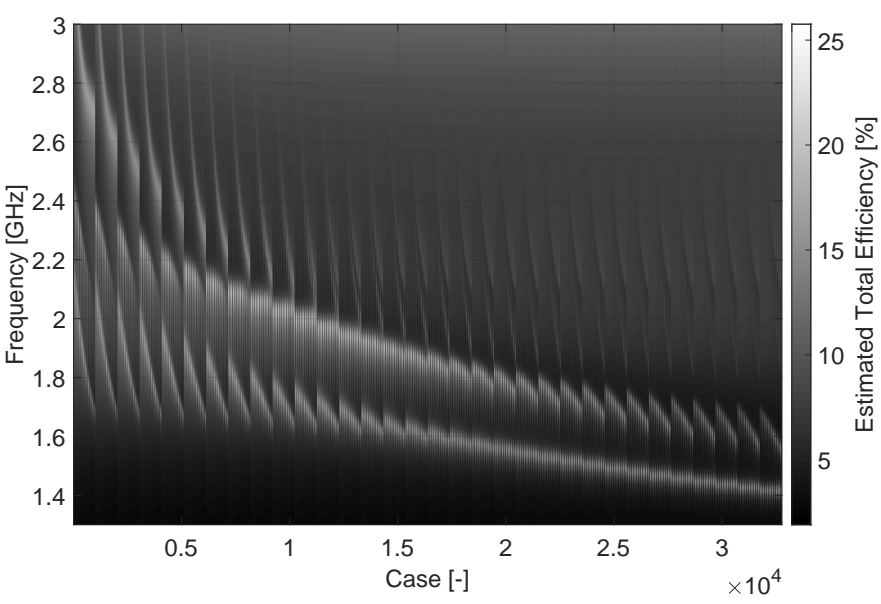

(b)

Fig. 7. The estimated (a) radiation and (b) total efficiencies of all calculated cases. In these figures, a lighter color corresponds to a higher efficiency. The worst radiation efficiencies tend to occur around the best matched frequencies (Fig. 6). Nonetheless, settings with an acceptable total efficiency can be found.

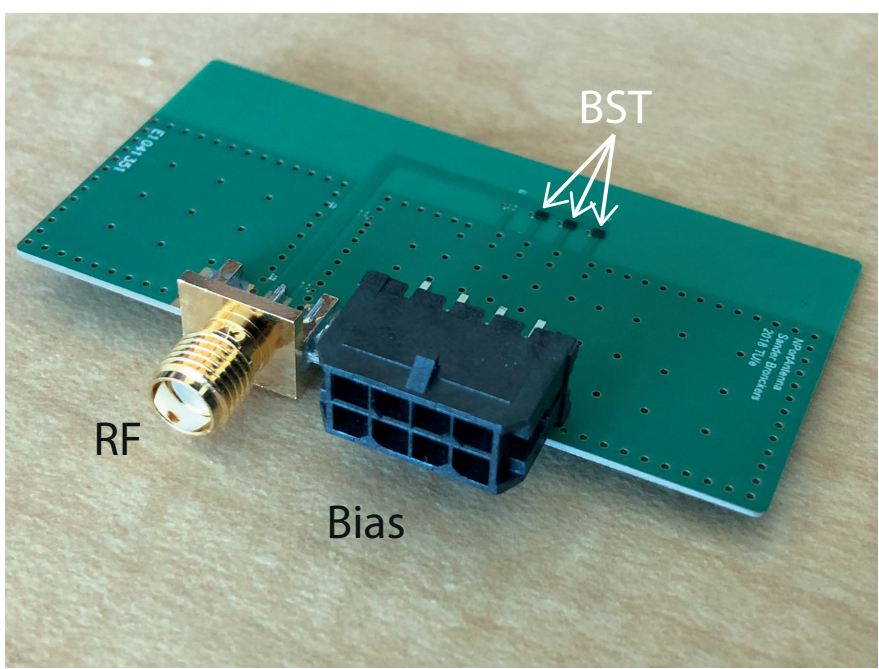

(a)

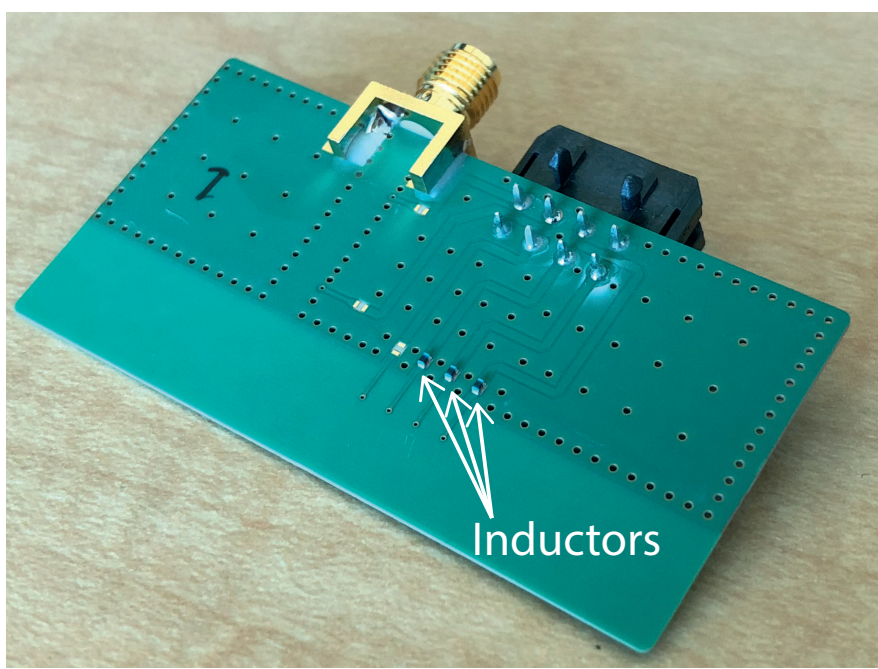

(b)

Fig. 8. The realized antenna (a) front and (b) back view. RF and bias connectors are indicated, as well as the locations of BST capacitors and inductors in the bias lines.

for the bias voltages are mounted. By using one cable for the bias connection, which will run along the coax cable, we ensure a tidy and repeatable connection and configuration.

\section{A. Measured Performance}

Before proceeding to the measured efficiencies, we have measured the antenna's reflection coefficients for the selected settings. Note that these settings were not selected for their input match, but rather for the estimated total efficiency. Therefore, we don't expect simulated and measured $\left|S_{11}\right|$ 's to be below e.g. $-10 \mathrm{~dB}$. We are simply verifying the predictive capabilities of the simulations. We have plotted the input reflection magnitude results in Fig. 9. There, we can observe that the match between simulations and measurements is nearly perfect around $2 \mathrm{GHz}$, the frequency where we estimated the model parameters for the BST components. For settings towards the lower end of the frequency range the difference in best-match frequency between simulation and measurement increases up to $70 \mathrm{MHz}$. This could be due to inaccuracies in the capacitor model, or to the bias voltages or deviations therein: for the lowest frequencies, the highest capacitance values are used, corresponding to the steepest slope in the voltage-capacitance relationship of the BST capacitors [45]. At low voltage the slope is approximately $-0.4 \mathrm{pF} / \mathrm{V}$, while for high voltages it decreases to $-0.02 \mathrm{pF} / \mathrm{V}$. Therefore the sensitivity of capacitance value to errors in the voltage is significantly higher for low voltages and high capacitance values. On the high frequency end the results also start to deviate, which is likely due to the capacitors being used close to the edge or outside of their recommended 0.7 to $2.7 \mathrm{GHz}$ operating range. In addition, as seen in the previous section, for the bands above $2.3 \mathrm{GHz}$ the center capacitor $\mathrm{C} 2$ is providing most of the total capacitance, as opposed to the frequencies below that. This can be observed in the different frequency 
TABLE I

OBTAINED SETTINGS ALONG WITH THEIR ESTIMATED EFFICIENCIES FOR FREQUENCIES SPACED $100 \mathrm{MHZ}$ APART. V1, V2 AND V3 DENOTE THE BIAS VOLTAGES FOR C1, C2 AND C3, RESPECTIVELY.

\begin{tabular}{|c|c|c|c|c|c|c|c|c|}
\hline $\begin{array}{c}\boldsymbol{F} \\
{[\mathbf{G H z}]}\end{array}$ & $\begin{array}{c}C 1 \\
{[\mathrm{pF}]}\end{array}$ & $\begin{array}{c}V 1 \\
{[\mathrm{~V}]}\end{array}$ & $\begin{array}{c}C 2 \\
{[\mathrm{pF}]}\end{array}$ & $\begin{array}{c}V 2 \\
{[\mathrm{~V}]}\end{array}$ & $\begin{array}{c}C 3 \\
{[\mathrm{pF}]}\end{array}$ & $\begin{array}{c}V 3 \\
{[\mathrm{~V}]}\end{array}$ & $\begin{array}{c}\eta_{\text {tot }} \\
{[\%]}\end{array}$ & $\begin{array}{c}\eta_{\text {rad }} \\
{[\%]}\end{array}$ \\
\hline $\mathbf{1 . 4}$ & 3.0 & 1.5 & 0.7 & 19.0 & 3.0 & 1.3 & 19 & 20 \\
\hline $\mathbf{1 . 5}$ & 2.6 & 2.3 & 0.7 & 19.0 & 2.6 & 2.1 & 19 & 21 \\
\hline $\mathbf{1 . 6}$ & 2.2 & 3.2 & 0.7 & 19.0 & 2.3 & 3.0 & 20 & 22 \\
\hline $\mathbf{1 . 7}$ & 1.9 & 4.1 & 0.7 & 19.0 & 2.1 & 3.6 & 21 & 27 \\
\hline $\mathbf{1 . 8}$ & 1.8 & 4.4 & 0.7 & 19.0 & 1.7 & 4.8 & 23 & 27 \\
\hline $\mathbf{1 . 9}$ & 1.6 & 5.6 & 0.7 & 19.0 & 1.6 & 5.6 & 25 & 32 \\
\hline $\mathbf{2 . 0}$ & 1.4 & 6.7 & 0.7 & 19.0 & 1.4 & 6.7 & 25 & 34 \\
\hline $\mathbf{2 . 1}$ & 1.4 & 6.7 & 0.7 & 19.0 & 1.2 & 8.7 & 24 & 30 \\
\hline $\mathbf{2 . 2}$ & 1.3 & 7.9 & 0.7 & 19.0 & 1.1 & 9.6 & 23 & 29 \\
\hline $\mathbf{2 . 3}$ & 1.1 & 9.6 & 0.7 & 19.0 & 1.0 & 10.7 & 22 & 33 \\
\hline $\mathbf{2 . 4}$ & 0.9 & 12.1 & 3.2 & 0.7 & 0.9 & 12.1 & 24 & 34 \\
\hline $\mathbf{2 . 5}$ & 0.9 & 13.9 & 3.0 & 1.3 & 0.9 & 13.9 & 23 & 35 \\
\hline $\mathbf{2 . 6}$ & 0.7 & 19.0 & 3.2 & 0.7 & 0.7 & 19.0 & 26 & 31 \\
\hline $\mathbf{2 . 7}$ & 0.7 & 19.0 & 2.6 & 2.1 & 0.7 & 19.0 & 24 & 32 \\
\hline $\mathbf{2 . 8}$ & 0.7 & 19.0 & 2.5 & 2.5 & 0.7 & 19.0 & 21 & 26 \\
\hline & & & & & & & & \\
\hline
\end{tabular}

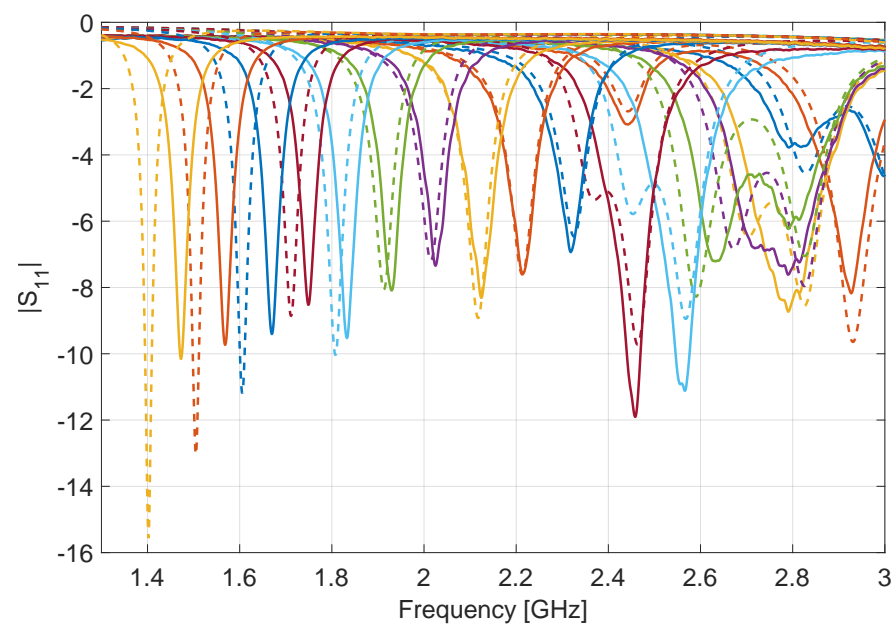

Fig. 9. Comparison of measured (solid) and simulated (dashed) input reflection magnitudes for selected cases. Different colors are used to distinguish between cases, and are kept the same for Fig. 9,10 and 13. The agreement is best around $2 \mathrm{GHz}$, where the BST capacitor models were determined.

behavior of the reflection coefficient, both in the simulations and the measurements. The input reflection results proved to be repeatable for different boards - therefore these differences are most likely repeatable within production series and, once known, could easily be compensated for using the tunability of the antenna.

The radiation and total efficiencies are measured in a reverberation chamber using the antenna-replacement method [59] with 100 paddle positions, $100 \mathrm{kHz}$ frequency sample spacing, and $5 \mathrm{MHz}$ frequency stirring. The chamber uses two rotating paddles, each of which are used in 10 positions, which are verified to have very low mutual correlations. No antenna position stirring was performed, as earlier experiments showed the chamber to have excellent spatial uniformity (with a standard deviation of the reference power transfer function smaller than $0.1 \mathrm{~dB}$ ). The results for both total and radiation efficiency are shown in Fig. 10. The estimated efficiencies, as given in Table I, are omitted here to be able to show the results clearly in a graph. The total efficiencies peak just below $15 \%$, while the estimated maximum was $25 \%$, and the radiation efficiencies are up to $50 \%$ (with estimates close to $100 \%$ ). This is to be expected, as the metallization and dielectric losses were not included in the estimate, creating an upper bound. As one would expect for a small antenna [5]-[9], the efficiency (both radiation and total) of the antenna decreases for decreasing frequency. The total efficiencies drop around $2.4 \mathrm{GHz}$ and $2.5 \mathrm{GHz}$, most likely due to a combination of a different mode being used (Table I) and non-ideal behavior of the capacitors that is unaccounted for in their circuit models. Comparing Fig. 9 with Fig. 10a, we can observe that, as expected, the radiation efficiency tends to drop close to the frequency where the antenna is matched. The change in the radiation efficiency for different cases also demonstrates that the radiation efficiency, in addition to input reflection, is indeed a critical parameter to estimate early in the design cycle. Nevertheless, our design procedure finds the radiating settings, as shown in Fig. 10b and predicted earlier in the maxima of the estimation in Fig. 7b. Moreover, the settings that were found show a smooth increase of maximum efficiency over frequency, as one would expect from the Chu-Harrington limit, suggesting a physical limitation. If cases were selected based on input matching only, this smooth increase would not be observed, instead showing large differences in efficiency between neighbouring frequencies. As will be discussed in the next subsection, the $50 \Omega$ reference impedance is a limiting factor for the total efficiency in this particular design, and results could be significantly improved when the antenna is connected to an adaptive impedance matching circuit, as is now common in most mobile applications [11]. It could also be improved by returning to step 1 and changing the geometrical design to include a matching network.

\section{B. Performance Connected to Adaptive Matching Network}

The challenge of achieving good input match and radiation efficiency at the same frequency is illustrated in Fig. 11, where we have combined the most crucial estimated and measured results for a single case. Note the great similarity in the frequency behavior of the estimated and measured efficiencies. From this figure it can be deduced that the total efficiency is, in fact, limited by the best input match occurring on the downward slope of the radiation efficiency. If the input match could be achieved at a slightly lower frequency, the total efficiency could be increased since the mismatch loss would be minimal at a frequency where the radiation efficiency is high. This suggests that the total efficiency may be improved by the addition of an adaptive matching circuit.

If we assume that the device the antenna will be connected to is not bound to $50 \Omega$, but has an adaptive matching circuit, the performance can indeed be improved. Such a network, 


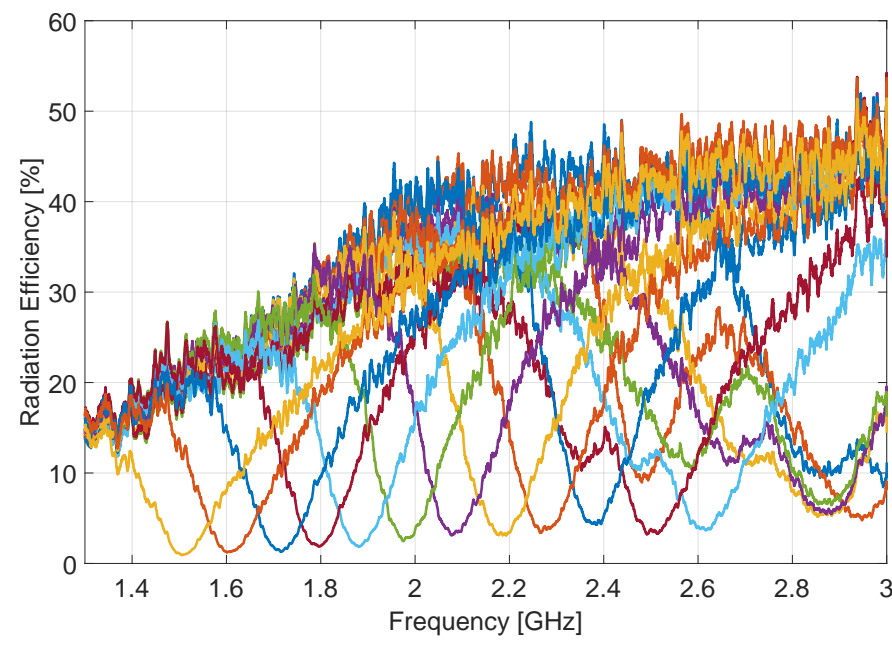

(a)

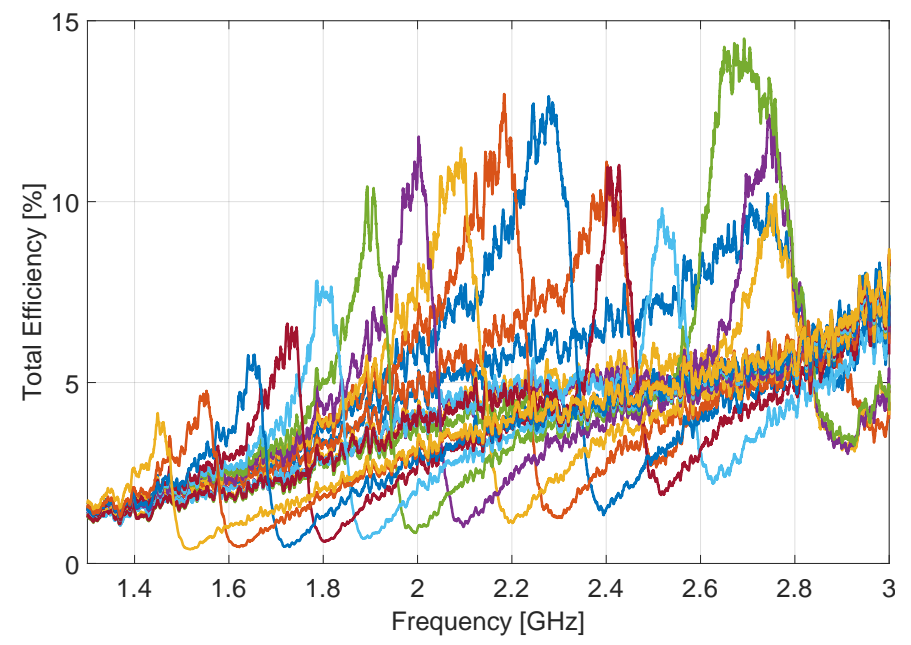

(b)

Fig. 10. Measured (a) radiation and (b) total efficiencies for selected cases. Different colors are used to distinguish between cases, and are kept the same for Fig. 9,10 and 13. There is a clear dip in both efficiencies at frequencies a bit above the maximum total efficiency and the minimum input reflection (Fig. 9).

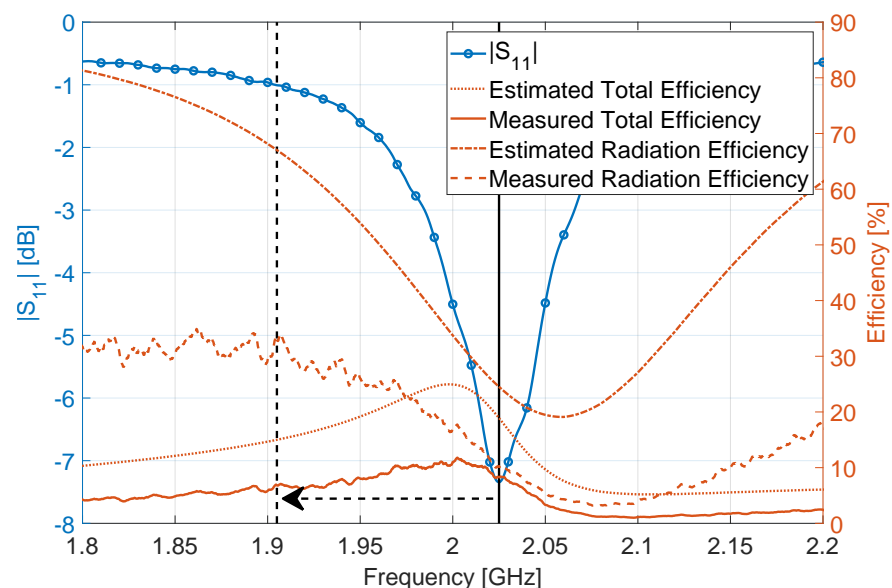

Fig. 11. Combined input reflection, total and radiation efficiencies (all measured) for a selected case. The input reflection is minimal at a frequency where the radiation efficiency is starting to dip. Therefore the total efficiency may be improved by shifting the minimum input reflection to a slightly lower frequency.

commonly present in modern smartphones, is connected to the antenna as shown in Fig. 12. Even in the simple case of a low-pass- $\pi$ matching network, using two of the same BST capacitors for $C_{\mathrm{M} 1}$ and $C_{\mathrm{M} 2}$ with a fixed $5.6 \mathrm{nH}$ inductor, the antenna's performance (as seen from the antenna-matching network interface) transforms to that shown in Fig. 13. The settings for the capacitors on the antenna itself are maintained. Since the adaptive matching network is not on the antenna and can be kept exceedingly small (it will most likely be integrated in a front-end module for a mobile device), it is assumed that the adaptive matching network is not radiating, only adapting the input match. In principle, the tuning possibilities of a traditional adaptive matching network are added to the tuning capabilities of the aperture-tuned antenna itself, which can be seen as a conventional antenna for each case from the adaptive matching network's point of view. Improving

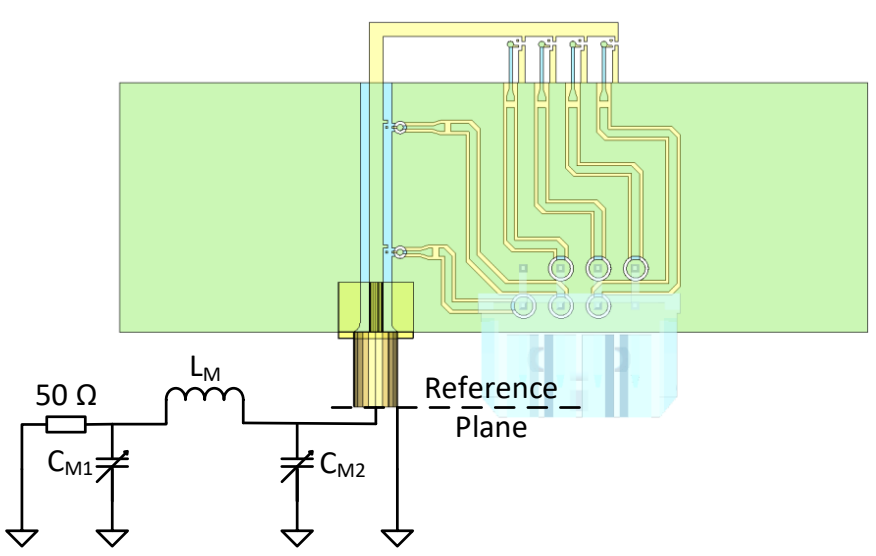

Fig. 12. The antenna's performance is projected (see Fig 13) with this lowpass pi matching network at the antenna connector interface.

the input match will increase the power flow through the tuning components in the aperture as well, but since these are operated in their linear region the increase in power loss in them will be proportional to the increase in the antenna's accepted power, thereby not affecting the radiation efficiency as the shapes of the current distributions in the aperture are not changed. The projected total efficiency is obtained by using the measured input impedance of the antenna, and then calculating the reflection coefficient from the adaptive matching circuit. The total efficiency is then calculated using this reflection coefficient and the measured radiation efficiency. Since the aperture of the antenna is not changed, its radiating performance remains constant, only improving the input match and thus increasing the magnitude of the radiated fields. After repeating this process for a set of possible settings for the adaptive matching circuit, the best total efficiency is selected and denoted as projected total efficiency. Note that this procedure is remarkably similar to the overall design method. Since the matching network itself is not radiating, and the reference plane is chosen between the matching network 


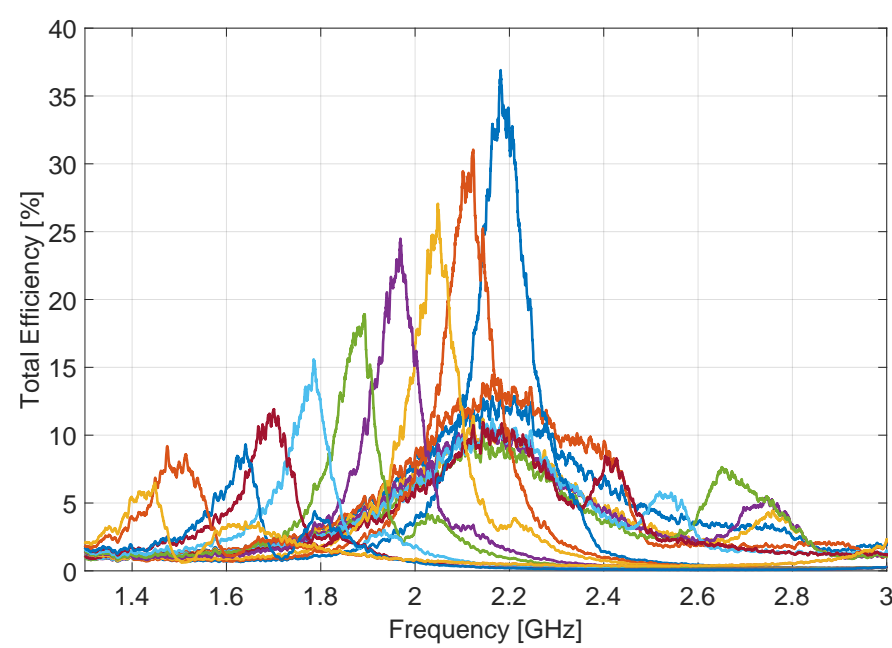

Fig. 13. Projected total efficiency from the interface with a low-pass pi adaptive matching network. Different colors are used to distinguish between cases, and are kept the same for Fig. 9,10 and 13. Using this network the performance up to $2.2 \mathrm{GHz}$ can be improved.

and the antenna, it is considered reasonable to simulate this part of the system. Due to the choice of matching network the performance above $2.2 \mathrm{GHz}$ is largely lost, but it demonstrates that, indeed, the performance can be improved by shifting the best input match and the worst radiation efficiency away from one another. A total efficiency of up to $35 \%$ at 2.2 $\mathrm{GHz}\left(\approx \frac{\lambda}{7}\right)$ can be achieved this way. Given the similarity of the process to obtain the projected total efficiency, this result would automatically be obtained if the additional matching network were included in the design of step 1, or even accounted for without being part of the simulated layout in step 3. Another way would be to require the antenna's input impedance to be within a pre-defined impedance region that can be matched, before selecting the case with the highest estimated radiation efficiency from the remaining cases.

Antenna designs incorporating high-Q MEMS tunable capacitors for mobile applications report total efficiencies between $-7 \mathrm{~dB}(20 \%)$ and $-2 \mathrm{~dB}(63 \%)$ in the $1.7-2.2 \mathrm{GHz}$ band [18] with a $10 \mathrm{~mm}$ long ILA design $\left(180 \mathrm{~mm}^{3}\right)$, and $55 \%$ at $1.85 \mathrm{GHz}$ for a $15 \mathrm{~mm}$ long design $\left(120 \mathrm{~mm}^{3}\right)$ [16]. Our design method, while employing a very simple and easily manufactured ILA of $20 \mathrm{~mm}$, arrives at similar results using tuning components with a much lower $\mathrm{Q}$, in a smaller volume $\left(50 \mathrm{~mm}^{3}\right)$, while it has a larger tuning range.

\section{CONCLUSION}

In this work, a new design method for frequencyreconfigurable antennas using multiple tuning components was proposed. The method aims to find the best tuning component settings given a geometrical design with possible locations from a total efficiency point of view, and could easily be adapted to include input match or radiation efficiency requirements. The design procedure was explained in detail, followed by a demonstration using an inverted-L antenna with three tunable BST capacitors, which showed satisfactory measured total efficiencies up to $35 \%$ when connected to an adaptive matching network, and radiation efficiencies up to $50 \%$, with a 1.4-2.8 GHz tuning range. It was demonstrated that the method can avoid cases (combinations of tuning component settings) that exhibit a good input match but a very low radiation efficiency in both simulations and measurements. This new method offers a time-efficient way to design frequencyreconfigurable antennas for maximum efficiency.

\section{REFERENCES}

[1] J. G. Andrews, S. Buzzi, W. Choi, S. V. Hanly, A. Lozano, A. C. K. Soong, and J. C. Zhang, "What will $5 \mathrm{~g}$ be?" IEEE Journal on Selected Areas in Communications, vol. 32, no. 6, pp. 1065-1082, Jun. 2014

[2] DMC R\&D Center Samsung Electronics Co., "5g vision," pp. 1-16, 2015. [Online].

[3] NGMN Alliance, "5g white paper," pp. 1-125, 2015. [Online].

[4] S. Bronckers, A. Roc'h, and B. Smolders, "Wireless receiver architectures towards 5g: Where are we?" IEEE Circuits and Systems Magazine, vol. 17, no. 3, pp. 6-16, Aug. 2017.

[5] H. Wheeler, "Fundamental limitations of small antennas," Proceedings of the IRE, vol. 35, no. 12, pp. 1479-1484, Dec. 1947.

[6] L. J. Chu, "Physical limitations of omni-directional antennas," Journal of Applied Physics, vol. 19, no. 12, p. 1163, 1948.

[7] R. F. Harrington, "Effect of antenna size on gain, bandwidth, and efficiency," Journal of Research of the National Bureau of Standards, Section D: Radio Propagation, vol. 64D, no. 1, p. 1, 1960.

[8] R. C. Hansen, "Fundamental limitations in antennas," Proceedings of the IEEE, vol. 69, no. 2, pp. 170-182, 1981.

[9] J. S. McLean, "A re-examination of the fundamental limits on the radiation q of electrically small antennas," IEEE Transactions on Antennas and Propagation, vol. 44, no. 5, p. 672, May 1996.

[10] C. A. Balanis, Modern Antenna Handbook, C. A. Balanis, Ed. Hoboken, NJ, USA: John Wiley \& Sons, Inc., Aug. 2008.

[11] J. L. Hilbert, Tunable RF Components and Circuits: Applications in Mobile Handsets. CRC Press, 2015.

[12] S. Yang, C. Zhang, H. Pan, A. Fathy, and V. Nair, "Frequencyreconfigurable antennas for multiradio wireless platforms," IEEE Microwave Magazine, vol. 10, no. 1, pp. 66-83, 2009.

[13] A. Petosa, "Frequency agile antennas for wireless communications - a survey," in 2010 14th International Symposium on Antenna Technology and Applied Electromagnetics \& the American Electromagnetics Conference. IEEE, Jul. 2010, pp. 1-4.

[14] C. G. Christodoulou, Y. A. Tawk, S. A. Lane, and S. R. Erwin, "Reconfigurable antennas for wireless and space applications," Proceedings of the IEEE, vol. 100, no. 7, pp. 2250-2261, Jul. 2012.

[15] A. Petosa, "An overview of tuning techniques for frequency-agile antennas," IEEE Antennas and Propagation Magazine, vol. 54, no. 5 , pp. 271-296, 2012.

[16] J. R. De Luis, A. S. Morris III, Q. Gu, and F. De Flaviis, "Tunable duplexing antenna system for wireless transceivers," IEEE Transactions on Antennas and Propagation, vol. 60, no. 11, pp. 5484-5487, 2012.

[17] N. Takemura, "Tunable inverted-l antenna with split-ring resonator structure for mobile phones," IEEE Transactions on Antennas and Propagation, vol. 61, no. 4, pp. 1891-1897, 2013.

[18] P. Bahramzy, O. Jagielski, S. Svendsen, and G. F. Pedersen, "Compact agile antenna concept utilizing reconfigurable front end for wireless communications," IEEE Transactions on Antennas and Propagation, vol. 62, no. 9, pp. 4554-4563, 2014.

[19] Y. A. Tawk, J. Costantine, and C. G. Christodoulou, "Cognitive-radio and antenna functionalities: A tutorial [wireless corner]," IEEE Antennas and Propagation Magazine, vol. 56, no. 1, pp. 231-243, Feb. 2014.

[20] A. S. Morris III, S. Caporal Del Barrio, V. Steel, and G. F. Pedersen, "Tunable antennas for mobile devices: Achieving high performance in compelling form factors," 2014 IEEE MTT-S International Microwave Symposium (IMS2014), vol. 1, pp. 1-4, 2014.

[21] J. Costantine, Y. A. Tawk, S. E. Barbin, and C. G. Christodoulou, "Reconfigurable antennas: design and applications," Proceedings of the IEEE, vol. 103, no. 3, pp. 424-437, 2015.

[22] A. P. Saghati, J. S. Batra, J. Kameoka, and K. Entesari, "Miniature and reconfigurable cpw folded slot antennas employing liquid-metal capacitive loading," IEEE Transactions on Antennas and Propagation, vol. 63, no. 9, pp. 3798-3807, Sep. 2015.

[23] Y. A. Tawk, J. Constantine, and C. G. Christodoulou, Antenna Design for Cognitive Radio. Norwood, MA: Artech House, 2016.

[24] K. Entesari and A. P. Saghati, "Fluidics in microwave components," IEEE Microwave Magazine, vol. 17, no. 6, pp. 50-75, Jun. 2016. 
[25] B. Majumder, K. Krishnamoorthy, J. Mukherjee, and K. P. Ray, "Frequency-reconfigurable slot antenna enabled by thin anisotropic double layer metasurfaces," IEEE Transactions on Antennas and Propagation, vol. 64, no. 4, pp. 1218-1225, 2016.

[26] A. Dey, R. Guldiken, and G. Mumcu, "Microfluidically reconfigured wideband frequency-tunable liquid-metal monopole antenna," IEEE Transactions on Antennas and Propagation, vol. 64, no. 6, pp. 2572 2576, 2016.

[27] S. Wang, L. Zhu, and W. Wu, "A novel frequency-reconfigurable patch antenna using low-loss transformer oil," IEEE Transactions on Antennas and Propagation, vol. 65, no. 12, pp. 7316-7321, 2017.

[28] L. Song, W. Gao, C. O. Chui, and Y. Rahmat-Samii, "Wideband frequency reconfigurable patch antenna with switchable slots based on liquid metal and 3-d printed microfluidics," IEEE Transactions on Antennas and Propagation, vol. 67, no. 5, pp. 2886-2895, 2019.

[29] C. N. Alvarez, R. Cheung, and J. S. Thompson, "Performance analysis of hybrid metal-graphene frequency reconfigurable antennas in the microwave regime," IEEE Transactions on Antennas and Propagation, vol. 65, no. 4, pp. 1558-1569, 2017.

[30] F. A. Ghaffar, M. Vaseem, L. Roy, and A. Shamim, "Design and fabrication of a frequency and polarization reconfigurable microwave antenna on a printed partially magnetized ferrite substrate," IEEE Transactions on Antennas and Propagation, vol. 66, no. 9, pp. 4866-4871, 2018.

[31] S. Soltani, P. Lotfi, and R. D. Murch, "A port and frequency reconfigurable mimo slot antenna for wlan applications," IEEE Transactions on Antennas and Propagation, vol. 64, no. 4, pp. 1209-1217, 2016.

[32] M. D. Wright, W. Baron, J. Miller, J. Tuss, D. Zeppettella, and M. Ali, "Mems reconfigurable broadband patch antenna for conformal applications," IEEE Transactions on Antennas and Propagation, vol. 66, no. 6, pp. 2770-2778, 2018.

[33] L. Ge, Y. Li, J. Wang, and C. Y. D. Sim, "A low-profile reconfigurable cavity-backed slot antenna with frequency, polarization, and radiation pattern agility," IEEE Transactions on Antennas and Propagation, vol. 65, no. 5, pp. 2182-2189, 2017.

[34] C. Gu, S. Gao, B. Sanz-Izquierdo, E. A. Parker, W. Li, X. Yang, and Z. Cheng, "Frequency-agile beam-switchable antenna," IEEE Transactions on Antennas and Propagation, vol. 65, no. 8, pp. 3819-3826, 2017.

[35] M. C. Tang, Z. Wen, H. Wang, M. Li, and R. W. Ziolkowski, "Compact, frequency-reconfigurable filtenna with sharply defined wideband and continuously tunable narrowband states," IEEE Transactions on Antennas and Propagation, vol. 65, no. 10, pp. 5026-5034, 2017.

[36] P. Lu, X. S. Yang, and B. Z. Wang, "A two-channel frequency reconfigurable rectenna for microwave power transmission and data communication," IEEE Transactions on Antennas and Propagation, vol. 65, no. 12, pp. 6976-6985, 2017.

[37] P. F. Hu, Y. M. Pan, and B. J. Hu, "Electrically small, planar, complementary antenna with reconfigurable frequency," IEEE Transactions on Antennas and Propagation, vol. PP, no. c, pp. 1-1, 2019.

[38] Cavendish Kinetics, "Product brief: Smartune antenna tuners," 2015.

[39] Q. Bai, R. Singh, K. L. Ford, T. O'Farrell, and R. J. Langley, "An independently tunable tri-band antenna design for concurrent multiband single chain radio receivers," IEEE Transactions on Antennas and Propagation, vol. 65, no. 12, pp. 6290-6297, 2017.

[40] S. C. Del Barrio, E. Foroozanfard, A. Morris, and G. F. Pedersen, "Tunable handset antenna: Enhancing efficiency on tv white spaces," IEEE Transactions on Antennas and Propagation, vol. 65, no. 4, pp. 2106-2111, 2017

[41] N. Nguyen-Trong, A. Piotrowski, and C. Fumeaux, "A frequencyreconfigurable dual-band low-profile monopolar antenna," IEEE Transactions on Antennas and Propagation, vol. 65, no. 7, pp. 3336-3343, 2017.

[42] P. F. Hu, Y. M. Pan, X. Y. Zhang, and B. J. Hu, "A filtering patch antenna with reconfigurable frequency and bandwidth using f-shaped probe," IEEE Transactions on Antennas and Propagation, vol. 67, no. 1, pp. 121-130, 2019.

[43] W. Gao, S. J. Chen, W. Withayachumnankul, and C. Fumeaux, "Horizontally polarized 360-degree beam-steerable frequency-reconfigurable antenna," IEEE Transactions on Antennas and Propagation, vol. PP, no. c, pp. 1-1, 2019.

[44] V. Steel and A. S. Morris III, "Tunable rf technology overview," Microwave Journal, pp. 1-7, 2012.

[45] ST Microelectronics, "Stptic-2712 parascan tunable integrated capacitor," 2015.

[46] S. Caporal Del Barrio, M. Pelosi, and G. F. Pedersen, "On the efficiency of frequency reconfigurable high-q antennas for $4 \mathrm{~g}$ standards," Electronics Letters, vol. 48, no. 16, p. 982, 2012.
[47] Y. Chen and D. Manteuffel, "A tunable decoupling and matching concept for compact mobile terminal antennas," IEEE Transactions on Antennas and Propagation, vol. 65, no. 4, pp. 1570-1578, 2017.

[48] J. M. Hannula, T. Saarinen, J. Holopainen, and V. Viikari, "Frequency reconfigurable multiband handset antenna based on a multichannel transceiver," IEEE Transactions on Antennas and Propagation, vol. 65, no. 9, pp. 4452-4460, 2017.

[49] N. Behdad and K. Sarabandi, "A varactor-tuned dual-band slot antenna," IEEE Transactions on Antennas and Propagation, vol. 54, no. 2, pp. 401-408, 2006

[50] — "Dual-band reconfigurable antenna with a very wide tunability range," IEEE Transactions on Antennas and Propagation, vol. 54, no. 2, pp. 409-416, Feb. 2006.

[51] V.-A. Nguyen, R. A. Bhatti, and S.-O. Park, "A simple pifa-based tunable internal antenna for personal communication handsets," IEEE Antennas and Wireless Propagation Letters, vol. 7, pp. 130-133, 2008.

[52] C. R. White and G. M. Rebeiz, "A shallow varactor-tuned cavity-backed slot antenna with a 1.9: 1 tuning range," Antennas and Propagation, IEEE Transactions on, vol. 58, no. 3, pp. 633-639, 2010.

[53] S. Zhu, D. G. Holtby, K. L. Ford, A. Tennant, and R. J. Langley, "Compact low frequency varactor loaded tunable srr antenna," IEEE Transactions on Antennas and Propagation, vol. 61, no. 4, pp. 23012304, 2013.

[54] P. Bahramzy, P. Olesen, P. Madsen, J. Bojer, S. Caporal Del Barrio, A. D. Tatomirescu, P. Bundgaard, A. S. Morris III, and G. F. Pedersen, "A tunable rf front-end with narrowband antennas for mobile devices," IEEE Transactions on Microwave Theory and Techniques, vol. 63, no. 10, pp. 3300-3310, Oct. 2015.

[55] P. Bahramzy, O. Jagielski, S. Svendsen, P. Olesen, and G. F. Pedersen, "Aspects of high-q tunable antennas and their deployment for $4 \mathrm{~g}$ mobile communications [antenna applications corner]," IEEE Antennas and Propagation Magazine, vol. 58, no. 4, pp. 70-81, Aug. 2016.

[56] L. A. Bronckers, A. Roc'h, and A. B. Smolders, "How tough are the front-end requirements for $4 \mathrm{~g}$-and-beyond handsets?" in 2017 47th European Microwave Conference (EuMC), vol. 0. IEEE, Oct. 2017, pp. 711-714.

[57] 3rd Generation Partnership Project; Technical Specification Group Radio Access Network, "Nr; user equipment (ue) radio transmission and reception; part 1: Range 1 standalone (release 15)," 2018.

[58] G. M. Rebeiz, K. Entesari, I. Reines, S.-j. Park, M. El-tanani, A. Grichener, and A. Brown, "Tuning in to rf mems," IEEE Microwave Magazine, vol. 10 , no. 6 , pp. $55-72$, Oct. 2009

[59] International Electrotechnical Commision, "Iec 61000-4-21:2011 electromagnetic compatibility (emc) - part 4-21: testing and measurement techniques - reverberation chamber test methods," 2011. [Online].

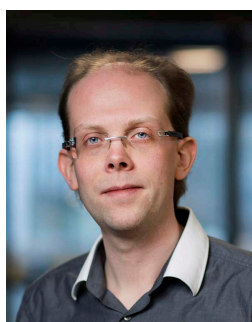

Laurens A. Bronckers received the M.Sc. degree (cum laude) in electrical engineering from Eindhoven University of Technology (TU/e) in 2015. After his M.Sc. he started his Ph.D. research in the same research group, focusing on frequencyreconfigurable antenna design for mobile applications and antenna measurement techniques. He was a guest researcher at the National Institute of Standards and Technology (NIST) for six months in 2018. He is currently with the Electromagnetics group of TU/e to pursue further advances in RF measurement techniques, with a focus on reverberation chamber methods. 


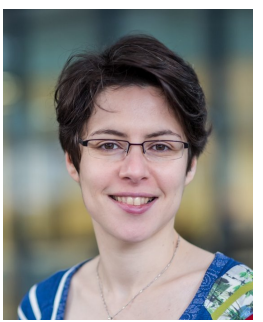

Anne Roc'h (M'07) received the M.Sc. degree in electronics and telecommunications from the Ecole Nationale d'Ingénieurs de Limoges, Limoges, France, in 2005 and the Ph.D. degree in electrical engineering from the University of Twente, Enschede, The Netherlands, in 2012. From June 2010 to March 2011, she was with the University of Eindhoven, Eindhoven, The Netherlands, where she worked on computational electromagnetics. She was an EMC expert from March 2011 to June 2014 in the industry. Since then, she has been an Assistant Professor with the University of Eindhoven in the field of Electromagnetic compatibility.

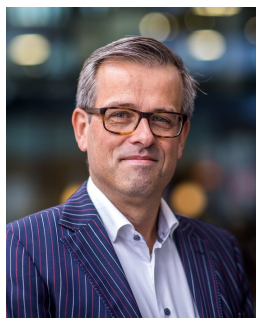

Bart Smolders was born in Hilvarenbeek, the Netherlands in 1965. He received his M.Sc. and $\mathrm{Ph} . \mathrm{D}$. degree in Electrical Engineering from the Eindhoven University of Technology (TU/e) in 1989 and 1994, respectively. From 1989 to 1991, he worked as an IC Designer at FEL-TNO, The Hague. From 1994 to 1997, he was a Radar System Designer with Thales, the Netherlands. From 1997 to 2000, he was project leader of the Square Kilometer Array (SKA) with the Netherlands Foundation for Research in Astronomy (ASTRON). From 2000 to 2010, he has been with NXP (formerly Philips) Semiconductors, The Netherlands, responsible for the innovation in the RF business line. Since 2010, he is a full-time professor at the TU/e in the Electromagnetics Group with special interest in antenna systems and applications. He currently leads several research projects in the area of integrated antenna systems operating at frequencies up to $120 \mathrm{GHz}$ for several application domains, including 5G/6G wireless communications, radar sensors and radio-astronomy. He is juniorpast chairman of the IEEE Benelux section and past-chair of the NERG (Nederlands Radio- en Elektronica Genootschap). He is Board member of the SWAN (Stichting Wetenschappelijke Activiteiten van het Nederlands URSI Committee) and member of the Advisory Board of ASTRON. Next to his research activities, he is the dean of the Electrical Engineering department of the TU/e. He has published more than 140 papers. 